\title{
Controle de Sistemas Variantes no Tempo: uma abordagem alternativa à técnica de controle via transformação Lyapunov-Floquet
}

\author{
Marco Antonio Travassos* Rodrigo Cardim* \\ Marcelo Carvalho Minhoto Teixeira* Edvaldo Assunção* \\ * UNESP - Universidade Estadual Paulista, Faculdade de Engenharia \\ de Ilha Solteira, Departamento de Engenharia Elétrica, Laboratório de \\ Pesquisa em Controle, Av. José Carlos Rossi, 1370, 15385-000, Ilha \\ Solteira, São Paulo, Brasil (e-mail: sr.marcotravassos@gmail.com, \\ rodrigo.cardim@unesp.br,marcelo.minhoto@unesp.br, \\ edvaldo.assuncao@unesp.br).
}

\begin{abstract}
It is known in the literature that the Lyapunov-Floquet transformation control technique can be applied in time-varying systems. However, the asymptotic stability of the system is not mathematically guaranteed. In this work the robust control theory is approached aiming its application in time-varying systems. This method is based on obtaining the representation of the system as a convex combination of the maximum and minimum of the variant terms of the matrices that determine the system. As will be seen, the robust control technique allows to obtain sufficient stability condition for linear time-varying systems, via LMIs. Finally, applications of this technique are presented in two systems, one linear and the other nonlinear.

Resumo: É conhecido na literatura que a técnica de controle via transformação LyapunovFloquet pode ser aplicada em sistemas variantes no tempo. Entretanto, a estabilidade assintótica do sistema não é garantida matematicamente. Neste trabalho é abordada a teoria de controle robusto visando sua aplicação em sistemas variantes no tempo. Este método se baseia em obter uma representação do sistema como uma combinação convexa dos máximos e mínimos dos termos variantes das matrizes que determinam o sistema. Como será visto, a técnica de controle robusto permite obter uma condição suficiente de estabilidade para sistemas lineares variantes, via LMIs. Por fim, são apresentadas aplicações desta técnica em dois sistemas, um linear e outro não linear.
\end{abstract}

Keywords: Time-variant systems; Linear Matrix Inequalities; Control Theory; Lyapunov-Floquet transformation; Rossler System.

Palavras-chaves: Sistemas variantes no tempo; Desigualdades Matriciais Lineares; Teoria de Controle; transformação Lyapunov-Floquet; Sistema de Rossler.

\section{INTRODUÇÃO}

Muitos problemas físicos e de engenharia são modelados por meio de sistemas variantes no tempo, por exemplo, oscilador de Duffing (Peruzzi, 2005), pêndulo simples excitado parametricamente (Mesquita, 2007), sistemas de captação de energia (Daqaq et al., 2009), etc. Para sistemas variantes no tempo, tanto a análise quanto o projeto do controlador são mais complexos, pois existe uma dependência explícita da variável temporal que requer um pouco mais de cuidado. Por exemplo, a condição de que os autovalores da matriz de um sistema linear e variante no tempo tenham parte real negativa, não é suficiente para garantir a estabilidade assintótica do sistema (Chen, 1998).

Para sistemas lineares variantes no tempo e periódicos, a estabilidade pode ser caracterizada em termos da Matriz de Transição de Estados (Sinha et al., 2000; Peruzzi et al., 2016).

Sinha et al. (2000) propuseram uma técnica de controle para sistemas variantes e periódicos, baseada na transformação Lyapunov-Floquet. Esta técnica foi aplicada em alguns sistemas variantes (Sinha et al., 2000; Kirkland and Sinha, 2016; Peruzzi et al., 2016). Entretanto, a estabilidade assintótica do sistema não é garantida matematicamente (Boghiu et al., 1998).

Este trabalho tem por objetivo abordar a Teoria de Controle Robusto aplicada aos sistemas lineares e não lineares variantes, como uma alternativa à técnica proposta por Sinha et al. (2000). Com a Teoria de Controle Robusto é possível obter uma lei de controle onde a estabilidade assintótica do sistema realimentado é garantida matematicamente. Por fim, são apresentadas aplicações no controle 
da Equação de Mathieu (sistema linear) e no sistema de Rossler (sistema não linear).

\section{SISTEMAS LINEARES VARIANTES NO TEMPO}

Um sistema linear variante no tempo é um sistema cuja representação em espaço de estados é dada por

$$
\dot{x}(t)=A(t) x(t)+B(t) u(t),
$$

sendo $x(t) \in \mathbb{R}^{n}$ o vetor de estados, $u(t) \in \mathbb{R}^{m}$ o vetor de controle, $A(t) \in \mathbb{R}^{n \times n}$ e $B(t) \in \mathbb{R}^{n \times m}$. Observe a dependência explícita da variável temporal nas matrizes $A(t)$ e $B(t)$.

Suponha que as matrizes $A(t)$ e $B(t)$ sejam dadas por $A(t)=\left[a_{i j}(t)\right]_{n \times n}$ e $B(t)=\left[b_{i j}(t)\right]_{n \times m}$ onde $a_{i j}(t)$ e $b_{i j}(t)$ representam os elementos da $i$-ésima linha e $j$-ésima coluna das matrizes $A(t)$ e $B(t)$, respectivamente. Pode ocorrer que nem todos os elementos da matriz $A(t)$ ou $B(t)$ sejam variantes. Considere ainda que os termos variantes sejam limitados. Seja $\left\{z_{1}(t), z_{2}(t), \ldots, z_{r}(t)\right\}$ uma enumeração dos termos que são variantes, tanto da matriz $A(t)$ quanto da matriz $B(t)$. O sistema (1) pode ser representado no formato de politopo dado por (Boyd et al., 1994)

$$
\dot{x}(t)=A(\alpha) x(t)+B(\alpha) u(t)
$$

onde

$$
\begin{aligned}
\alpha & =\left(\alpha_{1}, \ldots, \alpha_{N}\right), \\
\mathbb{K}_{N} & =\{1,2, \ldots, N\} \\
\Lambda_{N} & =\left\{\alpha \in \mathbb{R}^{N}: \sum_{i=1}^{N} \alpha_{i}=1, \alpha_{i} \geq 0, i \in \mathbb{K}_{N}\right\}, \\
A(\alpha) & =\sum_{i=1}^{N} \alpha_{i}(t) A_{i}, \alpha \in \Lambda_{N}, \\
B(\alpha) & =\sum_{i=1}^{N} \alpha_{i}(t) B_{i}, \alpha \in \Lambda_{N},
\end{aligned}
$$

sendo $N$ o número de vértices do politopo, dado por $N=2^{r}, r$ a soma dos números de termos variantes de $A(t)$ e $B(t), A_{i}$ e $B_{i}$ os vértices do politopo obtidos combinando os máximos e os mínimos dos termos variantes.

\subsection{Estabilidade e Taxa de Decaimento}

A seguir será apresentado uma condição suficiente para a estabilidade assintótica de sistemas lineares variantes no tempo.

Teorema 1. (Boyd et al., 1994) O ponto de equilíbrio $x^{*}=0$ do sistema

$$
\dot{x}(t)=A(\alpha) x(t)=\sum_{i=1}^{N} \alpha_{i}(t) A_{i} x(t),
$$

é assintoticamente estável se existe uma matriz $P$ definida positiva e simétrica tal que

$$
A_{i}^{\prime} P+P A_{i}<0, \forall i \in \mathbb{K}_{N} .
$$

Algumas vezes, além da estabilidade assintótica do sistema, pode-se estar interessado em melhorar o desempenho do sistema, com respostas mais rápidas. Para isso, devese levar em conta no projeto do controlador o conceito de taxa de decaimento.
Definição 1. A taxa de decaimento do sistema (1) não forçado $(u(t)=0)$ é definida como o maior escalar $\beta$ tal que

$$
\lim _{t \rightarrow+\infty} e^{\beta t}|| x(t) \|=0
$$

para toda trajetória $x(t) \in \mathbb{R}^{n}$.

Observação 1. Se a igualdade (3) é válida, então quanto maior for a taxa de decaimento $\beta$, mais rápido a norma $\|x(t)\|$ deverá convergir a zero.

A função de Lyapunov quadrática, dada por $V(x)=x^{\prime} P x$, pode ser usada para estabelecer um limitante inferior para a taxa de decaimento. Se a desigualdade

$$
\dot{V}(x(t)) \leq-2 \beta V(x(t)),
$$

for verificada para toda trajetória $x(t) \in \mathbb{R}^{n}$, então a taxa de decaimento do sistema (1) não forçado é no mínimo $\beta$ (Boyd et al., 1994).

Teorema 2. (Boyd et al., 1994) Uma condição equivalente a (4) é dada por

$$
A_{i}^{\prime} P+P A_{i}+2 \beta P \leq 0, \forall i \in \mathbb{K}_{N} .
$$

Observação 2. Em (5) fica garantido a estabilidade assintótica do sistema, pois como $P>0$, de (5) segue que $A_{i}^{\prime} P+P A_{i}<0, \forall i \in \mathbb{K}_{N}$.

\subsection{Projeto do Controlador}

Suponha que todos os estados estejam disponíveis e considere o vetor de controle $u(t)$, com um único ganho $K$, dado por

$$
u(t)=K x(t)
$$

O sistema (2) realimentado com a lei de controle (6) é descrito por

$$
\begin{aligned}
\dot{x}(t) & =[A(\alpha)+B(\alpha) K] x(t) \\
& =\sum_{i=1}^{N} \alpha_{i}(t)\left(A_{i}+B_{i} K\right) x(t) .
\end{aligned}
$$

O ganho $K$ deve ser determinado tal que o sistema (7) seja assintoticamente estável.

Teorema 3. (Boyd et al., 1994) Considere o sistema linear variante no tempo dado por (2). Se existem matrizes $W \in \mathbb{R}^{n \times n}, Z \in \mathbb{R}^{m \times n}$ e um escalar $\beta>0$ tais que

$$
\begin{aligned}
& W=W^{\prime}>0, \\
& W A_{i}^{\prime}+Z^{\prime} B_{i}^{\prime}+A_{i} W+B_{i} Z+2 \beta W \leq 0, \forall i \in \mathbb{K}_{N},
\end{aligned}
$$

então a lei de controle (6), com ganho $K=Z W^{-1}$, assegura a estabilidade assintótica do sistema (7) com taxa de decaimento $\beta$.

Prova. Por hipótese tem-se $W=W^{\prime}>0$ e para todo $i \in \mathbb{K}_{N}$ tem-se

$$
W A_{i}^{\prime}+Z^{\prime} B_{i}^{\prime}+A_{i} W+B_{i} Z+2 \beta W \leq 0 .
$$

Considerando $P=W^{-1}$ tem-se $K=Z P$ e, portanto, $Z=K P^{-1}$. Para todo $i \in \mathbb{K}_{N}$ tem-se 


$$
\begin{aligned}
& P^{-1} A_{i}^{\prime}+\left(K P^{-1}\right)^{\prime} B_{i}^{\prime}+A_{i} P^{-1}+B_{i}\left(K P^{-1}\right) \\
& +2 \beta P^{-1} \leq 0, \\
\Rightarrow & P^{-1}\left(A_{i}+B_{i} K\right)^{\prime}+\left(A_{i}+B_{i} K\right) P^{-1} \\
& +2 \beta P^{-1} \leq 0, \\
\Rightarrow & P^{-1} \sum_{i=1}^{N} \alpha_{i}\left(A_{i}+B_{i} K\right)^{\prime}+\sum_{i=1}^{N} \alpha_{i}\left(A_{i}+B_{i} K\right) P^{-1} \\
& +2 \beta P^{-1} \leq 0 .
\end{aligned}
$$

Pré e pós multiplicando a desigualdade acima por $P$, segue que

$$
\begin{aligned}
& (A(\alpha)+B(\alpha) K)^{\prime} P+P(A(\alpha)+B(\alpha) K) \\
& +2 \beta P \leq 0 \\
\Rightarrow & x^{\prime}\left[(A(\alpha)+B(\alpha) K)^{\prime} P+P(A(\alpha)+B(\alpha) K)\right. \\
& +2 \beta P] x \leq 0, \\
\Rightarrow & ((A(\alpha)+B(\alpha) K) x)^{\prime} P x+x^{\prime} P(A(\alpha)+B(\alpha) K) x \\
& +2 \beta x^{\prime} P x \leq 0, \\
\Rightarrow & \dot{V}(x) \leq-2 \beta V(x) .
\end{aligned}
$$

Neste artigo, para solução das Desigualdades Matriciais Lineares (LMIs, do inglês Linear Matrix Inequalities) dadas por (8), foram utilizados o software MatLab ${ }^{\circledR}$ juntamente com o solver "SeDuMi" (Sturm, 1999) e a toolbox "YALMIP"(Löfberg, 2004).

\subsection{Equação de Mathieu}

A técnica de controle, descrita na subseção anterior, foi aplicada no controle da equação de Mathieu (Sinha and Butcher, 1997) dada por

$$
\ddot{y}(t)+(a+b \cos (t)) y(t)=u(t) .
$$

Considerado as seguintes mudanças de variáveis $x_{1}=y$ e $x_{2}=\dot{y}$, obtém-se a representação em espaço de estados da equação acima, dada por

$$
\left[\begin{array}{l}
\dot{x}_{1} \\
\dot{x}_{2}
\end{array}\right]=\left[\begin{array}{cc}
0 & 1 \\
-(a+b \cos (t)) & 0
\end{array}\right]\left[\begin{array}{l}
x_{1} \\
x_{2}
\end{array}\right]+\left[\begin{array}{l}
0 \\
1
\end{array}\right] u .
$$

Seja $A_{21}(t)=-(a+b \cos (t))=-a-b \cos (t)$ e suponha que $b \geq 0$. Neste caso, pode-se mostrar que $-a-b \leq A_{21}(t) \leq$ $-\bar{a}+b$. Observe que o número de termos variantes é 1 . Logo, o número de vértices do politopo será $N=2^{1}=2$. O sistema (9) pode ser escrito como

$$
\dot{x}(t)=\sum_{i=1}^{2} \alpha_{i}(t) A_{i} x(t)+\sum_{i=1}^{2} \alpha_{i}(t) B_{i} u(t)
$$

sendo

$$
\begin{aligned}
& A_{1}=\left[\begin{array}{cr}
0 & 1 \\
-a-b & 0
\end{array}\right] ; A_{2}=\left[\begin{array}{cr}
0 & 1 \\
-a+b & 0
\end{array}\right] ; \\
& B_{1}=B_{2}=\left[\begin{array}{l}
0 \\
1
\end{array}\right] .
\end{aligned}
$$

Inicialmente, o sistema (9) foi simulado em malha aberta, isto é, $u(t)=0$. Foram considerados os seguintes valores de parâmetros: $a=0.5$ e $b=1$. A resposta das variáveis de estado estão exibidas no gráfico a seguir. Para as simulações foi considerado a condição inicial $x_{0}=[4-3]^{\prime}$.

Na Figura 1 percebe-se o comportamento não assintótico das variáveis de estado, aumentando suas amplitudes a medida que o tempo cresce.
Figura 1. variáveis de estado em malha aberta

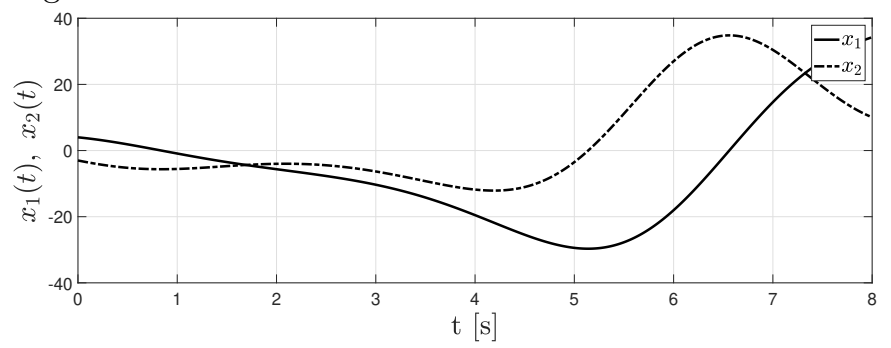

Primeiramente, o ganho $K$ foi projetado para estabilizar o sistema conforme o Teorema 3 e sem taxa de decaimento, isto é, $\beta=0$. A matriz $P$ e o ganho $K$ obtidos via LMIs foram:

$$
P=\left[\begin{array}{ll}
3.3015 & 0.8842 \\
0.8842 & 1.0257
\end{array}\right] ; K=\left[\begin{array}{l}
-3.8010 \\
-1.7398
\end{array}\right]^{\prime}
$$

As Figuras 2 exibe o comportamento das variáveis de estado do sistema controlado, considerando a mesma condição inicial $x_{0}=\left[\begin{array}{cc}4 & -3\end{array}\right]^{\prime}$. A Figura 3 exibe o comportamento do sinal de controle $u(t)$.

Figura 2.variáveis de estado do sistema controlado

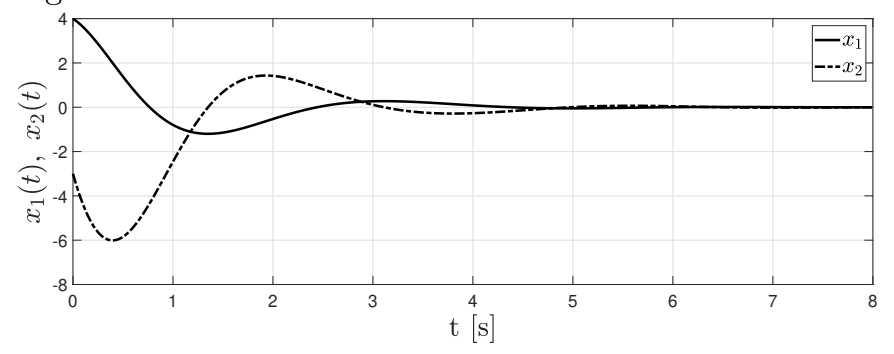

Figura 3. sinal de controle

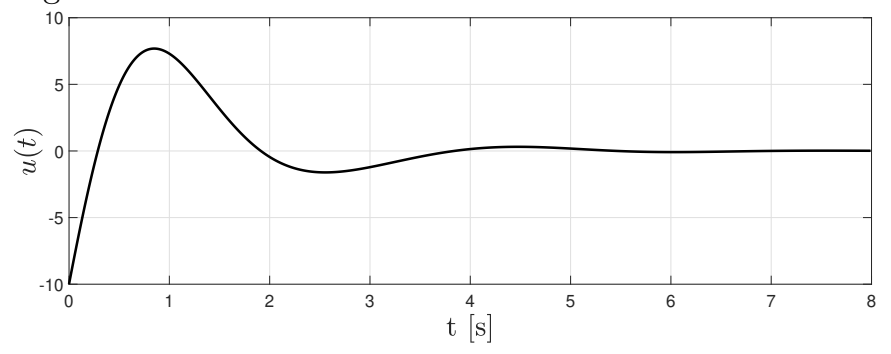

Pode-se perceber que o controlador foi eficiente para controlar o sistema, com um transitório de aproximadamente 6 segundos.

Com intuito de melhorar a resposta do sistema, foi projetado um controlador considerando também a seguinte taxa de decaimento $\beta=1$, conforme Teorema 3. A matriz $P$ e o ganho $K$ obtidos via LMIs foram:

$$
P=\left[\begin{array}{cc}
10.9711 & 2.5191 \\
2.5191 & 1.1274
\end{array}\right] ; K=\left[\begin{array}{l}
-15.7837 \\
-5.1598
\end{array}\right]^{\prime} .
$$

As Figuras 4 e 5 exibem as variáveis de estado e o sinal de controle $u(t)$ do sistema controlado, com taxa de decaimento $\beta=1$, respectivamente.

Percebe-se que, com a taxa de decaimento, obteve-se uma resposta mais rápida com transitório de aproximadamente 3 segundos. Entretanto, obteve-se também um aumento 
considerável da amplitude do controlador, que passou de 10 para 50, em módulo.

Figura 4. variáveis de estado da equação de Mathieu

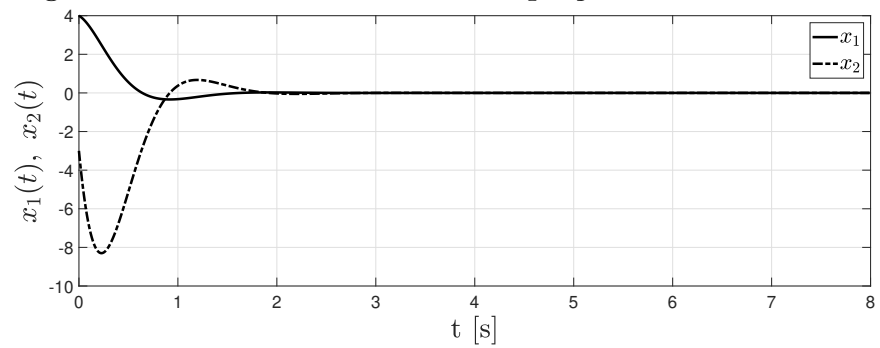

Figura 5. sinal de controle

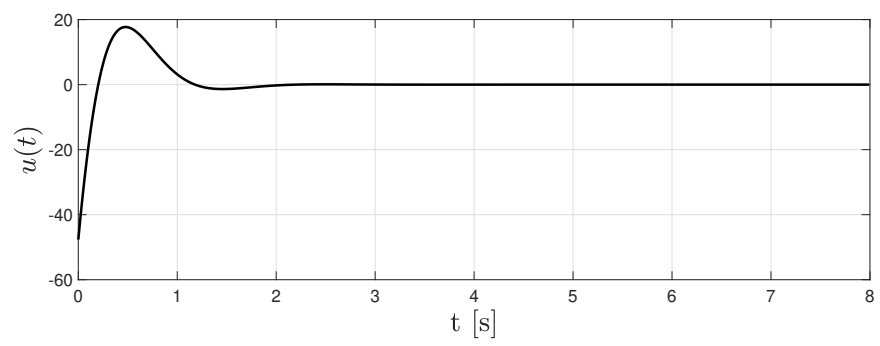

\section{CONTROLE DE SISTEMAS NÃO LINEARES: TRAJETÓRIA PREDEFINIDA}

Nesta seção, será ilustrado como a Teoria de Controle Robusto pode ser aplicada em sistemas não lineares, com o objetivo de obter uma lei de controle que conduza a trajetória do sistema realimentado para uma trajetória predefinida.

Para iniciar a discussão, suponha que a equação dinâmica de um sistema dinâmico variante no tempo seja dada por

$$
\dot{x}(t)=f(t, x(t))+B(t) u(t),
$$

sendo $x(t) \in \mathbb{R}^{n}$ o vetor de estados, $u(t) \in \mathbb{R}^{m}$ o vetor de controle, $f(t, x) \in \mathbb{R}^{n}$ e $B(t) \in \mathbb{R}^{n \times m}$. Suponha que se deseja conduzir a trajetória do sistema controlado $x(t)$ para uma trajetória predefinida $y(t)$. Dado que a trajetória desejada $y(t)$ e a equação dinâmica do sistema $f(t, x)$ são conhecidas, pode-se considerar a seguinte equação dinâmica

$$
\dot{x}(t)=f(t, x(t))+\bar{u}(t),
$$

onde a lei de controle $\bar{u}(t)$ é dada por

$$
\bar{u}(t)=u_{f}(t)+B(t) u(t),
$$

sendo

$$
u_{f}(t)=\dot{y}(t)-f(t, y(t)) .
$$

A parcela $u_{f}(t)$ do controlador $\bar{u}(t)$ é chamada de feedforward e a parcela $B(t) u(t)$ de feedback. Suponha ainda que todos os estados estejam disponíveis, então pode-se considerar a seguinte lei de controle para a parcela feedback $u(t)$, dada por

$$
u(t)=K(t)(x(t)-y(t)) .
$$

A matriz $K(t)$ é chamada de ganho de feedback do controlador. Substituindo (14) e (15) em (13) obtém-se

$$
\dot{x}(t)-\dot{y}(t)=f(t, x(t))-f(t, y(t))+B(t) u(t) .
$$

Definindo o erro dinâmico entre $x(t)$ (trajetória do sistema controlado) e $y(t)$ (trajetória desejada) como sendo

$$
e(t)=x(t)-y(t)
$$

tem-se

$$
\dot{e}(t)=\dot{x}(t)-\dot{y}(t)
$$

Considere a função definida por

$$
g(t, e(t))=f(t, e(t)+y(t))-f(t, y(t))
$$

Substituindo as três últimas igualdades acima em (16) obtém-se a equação do erro dinâmico entre a trajetória do sistema controlado e a trajetória desejada, dada por

$$
\dot{e}(t)=g(t, e(t))+B(t) u(t) \text {. }
$$

Defina a seguinte matriz

$$
A(t)=\left[\frac{\partial g_{j}}{\partial e_{i}}(t, 0)\right]_{n \times n} .
$$

Se a condição

$$
\lim _{\|e\| \rightarrow 0} \sup _{t \geq 0} \frac{\|g(t, e)-A(t) e+B(t) u(t)\|}{\|e\|}=0,
$$

for verificada, então o sistema

$$
\dot{e}(t)=A(t) e(t)+B(t) u(t)
$$

é chamado de linearização do sistema (18) ao redor do ponto de equilíbrio $e=0$ (Sinha et al., 2000; Slotine et al., 1991). O sistema (19) é um sistema linear variante no tempo. Em seu artigo, Sinha et al. (2000) propôs obter a lei de controle $u(t)$ satisfazendo a seguinte igualdade

$$
Q(t)^{-1} B(t) u(t)=B_{0} F_{0} Q(t)^{-1} e(t),
$$

onde $Q(t)^{-1}$ é a inversa da transformação LyapunovFloquet, e $B_{0}, F_{0}$ são matrizes conveniente e de dimensões adequadas. Infelizmente, na maioria dos casos não é possível obter uma lei de controle satisfazendo a equação acima e, por isso, uma lei de controle $u(t)$ aproximada é considerada, dada por

$$
u(t)=B(t)^{*} Q(t) B_{0} F_{0} Q(t)^{-1} e(t),
$$

onde $B(t)^{*}$ é a matriz inversa generalizada da matriz $B(t)$, dada por

$$
B(t)^{*}=\left(B(t)^{\prime} B(t)\right)^{-1} B(t) .
$$

Como uma lei de controle aproximada é utilizada, a estabilidade assintótica do sistema linearizado não é garantida matematicamente (Boghiu et al., 1998).

Neste trabalho é proposto aplicar a técnica descrita na Seção 2 para o sistema linearizado, a fim de obter a lei de controle $u(t)$ tal que o sistema (19) seja assintoticamente estável, isto é, $e(t) \rightarrow 0$ quando $t \rightarrow \infty$. Consequentemente, $x(t) \rightarrow y(t)$ quando $t \rightarrow \infty$ como desejado.

Como foi visto na Seção 2, a técnica descrita nesta seção garante matematicamente a estabilidade assintótica do sistema realimentado (19). Além disso, observe que a transformação Lyapunov-Floquet $Q(t)$ aparece na lei de controle (20), assim para simular tal lei de controle é preciso recorrer a métodos numéricos, como por exemplo o método proposto por Sinha and Butcher (1997).

\subsection{Sistema de Rossler}

A fim de testar a eficiência do método descrito na Seção 3 , considere o seguinte sistema de equações diferenciais ordinárias dadas por

$$
\left\{\begin{array}{l}
\dot{x}_{1}=-x_{2}-x_{3}, \\
\dot{x}_{2}=x_{1}+a x_{2}, \\
\dot{x}_{3}=b+x_{3}\left(x_{1}-c\right) .
\end{array}\right.
$$

O sistema (21) é não linear e é chamado de sistema de Rossler. Para o conjunto de parâmetros $a=0.2$, 
$b=0.2$ e $c=5.7$, o sistema acima possui um atrator caótico conhecido como Rossler Bands (Peruzzi, 2005). Esta sistema foi simulado considerando a condição incial $x_{0}=\left[\begin{array}{lll}4 & -3 & 1\end{array}\right]^{\prime}$. Na Figura 6 é exibido o comportamento das variáveis de estado do sistema de Rossler. A Figura 7 exibe a Rossler bands no plano de fase.

Figura 6. variáveis de estado do sistema de Rossler em

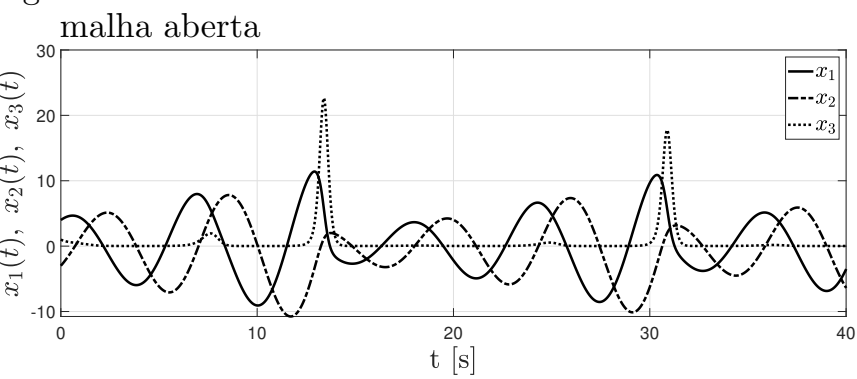

Figura 7. plano de fase do sistema de Rossler em malha aberta

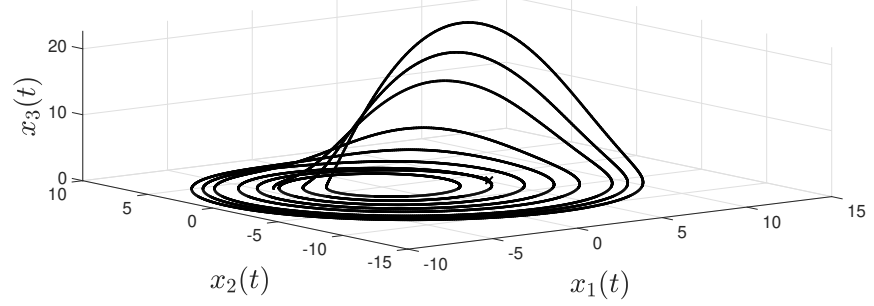

O controle linear apresentado na Seção 3 pode ser usado para conduzir o comportamento caótico do sistema de Rossler para uma orbita desejada. Considere $f_{1}(t, x), f_{2}(t, x)$ e $f_{3}(t, x)$ definidas da seguinte forma

$$
\begin{aligned}
& f_{1}(t, x)=-x_{2}-x_{3}, \\
& f_{2}(t, x)=x_{1}+a x_{2}, \\
& f_{3}(t, x)=b+x_{3}\left(x_{1}-c\right) c .
\end{aligned}
$$

Se $f(t, x)=\left[\begin{array}{lll}f_{1}(t, x) & f_{2}(t, x) & f_{3}(t, x)\end{array}\right]^{\prime}$, então

$$
\dot{x}=f(t, x)+u(t),
$$

sendo $u(t)=u_{f}(t)+u_{t}(t)$ o sinal de controle que é a soma da parcela feedforward e feedback, sendo que a parcela feedback é dada por $u_{t}(t)=B u_{b}(t)$, onde $B=\left[\begin{array}{ccc}1 & 0 & 0\end{array}\right]^{\prime}$. Deseja-se obter uma lei de controle $u(t)$ que conduza a trajetória caótica do sistema de Rossler para uma órbita desejada $y(t)$ dada por

$$
y(t)=\left[\begin{array}{lll}
2 \cos (t) & 2 \operatorname{sen}(t) & 0
\end{array}\right]^{\prime} .
$$

A seguir, a parcela feedforward $u_{f}(t)$ e a parcela feedback $u_{t}(t)$ do controlador serão determinadas. Conforme a equação (15), a parcela feedforward é dada por

$$
\begin{aligned}
u_{f}(t) & =\dot{y}(t)-f(t, y) \\
& =\left[\begin{array}{c}
-2 \operatorname{sen}(t) \\
2 \cos (t) \\
0
\end{array}\right]-\left[\begin{array}{c}
2 \cos (t)+0,2 \times 2 \operatorname{sen}(t) \\
0,2
\end{array}\right] \\
& =\left[\begin{array}{c}
0 \\
-0,4 \operatorname{sen}(t) \\
-0,2
\end{array}\right] .
\end{aligned}
$$

Substituindo (23) em (22) e considerando $e(t)=x(t)-y(t)$ obtém-se a equação do erro dinâmico entre a trajetória do sistema e a trajetória desejada, dada por

$$
\dot{e}(t)=g(t, e(t))+u_{t}(t)
$$

sendo $g(t, e(t))$ dada por (17). A linearização deste sistema ao redor do ponto $e=0$ é dada por

$$
A(t)=\left[\begin{array}{ccc}
0 & -1 & -1 \\
1 & 0.2 & 0 \\
0 & 0 & -5.7+2 \operatorname{sen}(t)
\end{array}\right]
$$

Portanto, a equação dinâmica do modelo linearizado é dada por

$$
\dot{e}(t)=\left[\begin{array}{ccc}
0 & -1 & -1 \\
1 & 0.2 & 0 \\
0 & 0 & -5.7+2 \operatorname{sen}(t)
\end{array}\right] e(t)+\left[\begin{array}{l}
1 \\
0 \\
0
\end{array}\right] u_{b}(t) .
$$

O sistema linearizado é um sistema linear e variante no tempo e, portanto, a técnica descrita na Seção 2 será usada para obter a parcela feedback $u_{b}(t)$ do controlador. Considere $A_{33}(t)=-5.7+2 \operatorname{sen}(t)$. Pode-se mostrar que $-7.7 \leq A_{33}(t) \leq-3.7$. Observe que o número de vértices do politopo é $\bar{N}=2$. Assim, o sistema linearizado acima pode ser escrito como

$$
\dot{e}(t)=\sum_{i=1}^{2} \alpha_{i}(t) A_{i} e(t)+\sum_{i=1}^{2} \alpha_{i}(t) B_{i} u_{b}(t),
$$

sendo

$$
\begin{aligned}
& A_{1}=\left[\begin{array}{ccc}
0 & -1 & -1 \\
1 & 0.2 & 0 \\
0 & 0 & -7.7
\end{array}\right] ; A_{2}=\left[\begin{array}{ccc}
0 & -1 & -1 \\
1 & 0.2 & 0 \\
0 & 0 & -3.7
\end{array}\right] \\
& B_{1}=B_{2}=\left[\begin{array}{lll}
1 & 0 & 0
\end{array}\right]^{\prime}
\end{aligned}
$$

O ganho $K$ projetado para estabilizar o sistema linearizado com taxa de decaimento $\beta=1$ foi obtido de acordo com o Teorema 3 . As matrizes $P$ e $K$ obtidas foram:

$$
P=\left[\begin{array}{lll}
1.1375 & 2.1173 & 0.0000 \\
2.1173 & 6.2191 & 0.0000 \\
0.0000 & 0.0000 & 6.5999
\end{array}\right] ; K=\left[\begin{array}{r}
-4.5742 \\
-8.8863 \\
1.0000
\end{array}\right]^{\prime}
$$

Novamente, $x_{0}=\left[\begin{array}{lll}4 & -3 & 1\end{array}\right]^{\prime}$ foi considerada como condição inicial . As Figuras 8 a 10 exibem o comportamento das variáveis de estado juntamente com a respectiva trajetória desejada.

Figura 8. estado controlado $x_{1}$ e desejado $y_{1}$

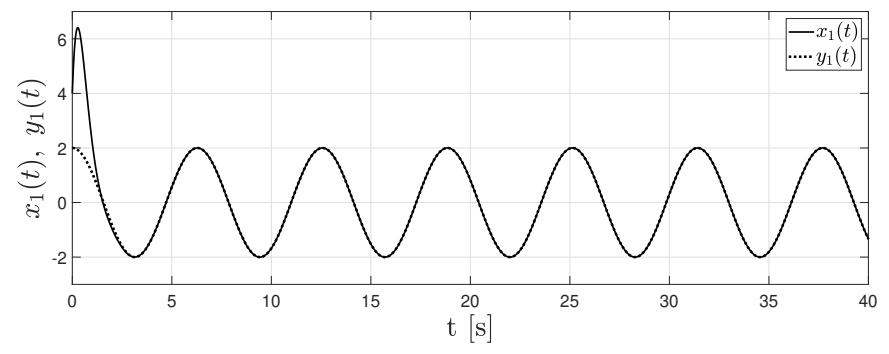

Figura 9. estado controlado $x_{2}$ e desejado $y_{2}$

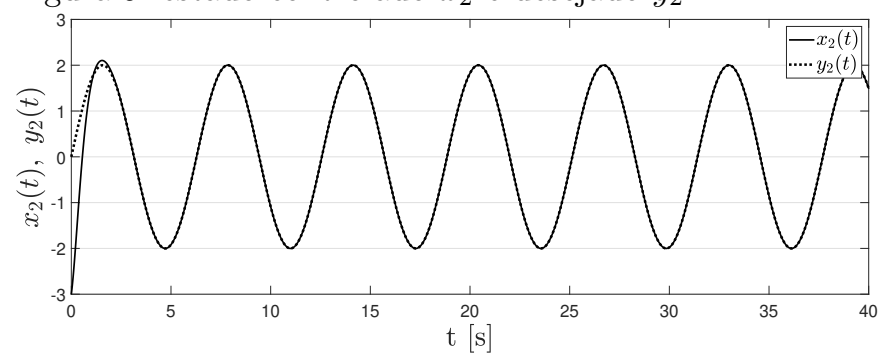


Figura 10. estado controlado $x_{3}$ e desejado $y_{3}$

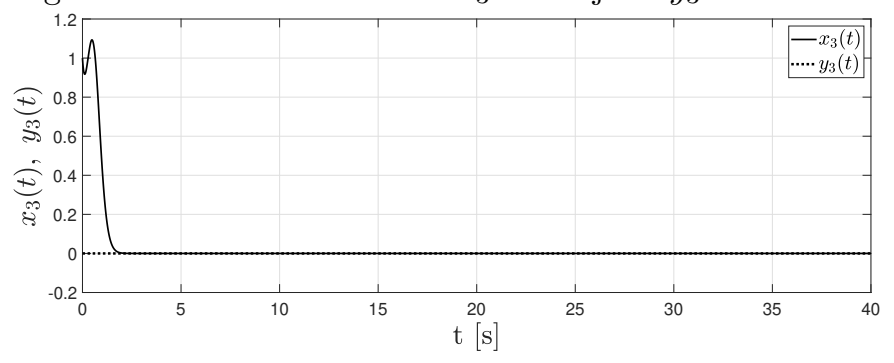

Percebe-se que a técnica de controle foi eficiente para conduzir as variáveis de estado do sistema controlado para os estados predefinidos.

A Figura 11 exibe o plano de fase do sistema de Rossler controlado. Observe que o controlador foi eficiente para conduzir a trajetória caótica para a trajetória predefinida, mostrando assim a eficiência da técnica de controle.

Figura 11. plano de fase do sistema de Rossler controlado x condic̃ao inicial一órbita desejada-órbita controlada

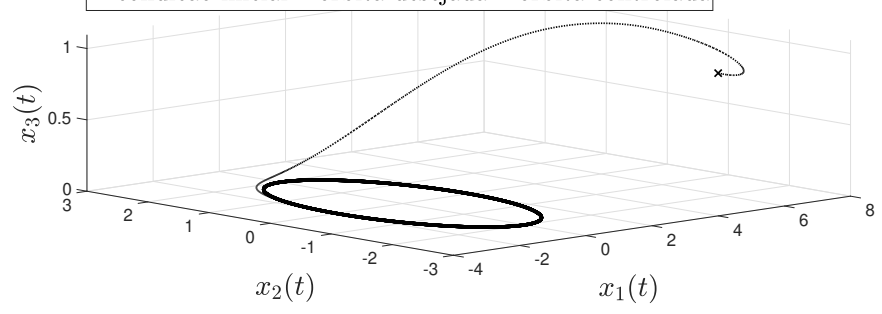

A Figura 12 exibe o comportamento da parte feedback $u_{b}(t)$ do controlador. Observe que o controlador atua de forma contínua e periódica para levar a trajetória controlada para a trajetória desejada.

Figura 12. parte feedback do sinal de controle

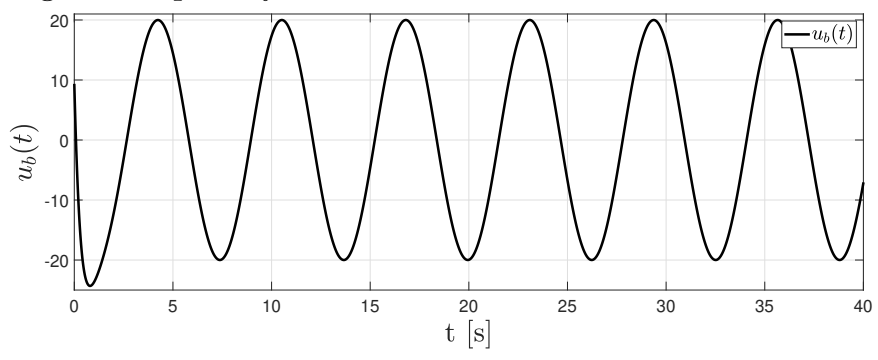

Observação 3. Veja que o sistema (21) não é variante no tempo, entretanto, quando se considere a equação do erro dinâmico entre a trajetória do sistema e a predefinida obtém-se um sistema variante no tempo (ver (24)). Isso mostra que a técnica de controle descrita na Seção 3 também pode ser aplicada para sistemas não lineares invariantes, a fim de conduzir a trajetória do sistema para uma predefinida.

\section{CONCLUSÕES}

Neste trabalho pode-se testificar que a Teoria de Controle Robusto pode ser usada para controlar sistemas variantes no tempo, tanto sistemas lineares quanto não lineares.
Neste último caso deve-se trabalhar com o erro dinâmico entre a trajetória controlada e a desejada. Além disso, a vantagem de se usar a Teoria de Controle Robusto, em relação a técnica proposta por Sinha et al. (2000) é que, para o sistema linear, existem condições LMIs suficientes que garantem a estabilidade quadrática assintótica do sistema. Além disso, a técnica via transformação LyapunovFloquet é restrita apenas aos sismas variantes no tempo e periódico, enquanto que a técnica via controle robusto pode ser aplicada em sistemas que não são periódicos, e também não precisar recorrer a métodos numéricos para obter a transformação Lyapunov-Floquet.

\section{AGRADECIMENTOS}

O presente trabalho foi realizado com apoio da Coordenação de Aperfeiçoamento de Pessoal de Nível Superior Brasil (CAPES) - Código de Financiamento 001. Os autores também agradecem as agências de fomento FAPESP e CNPq.

\section{REFERÊNCIAS}

Boghiu, D., Sinha, S., and Marghitu, D. (1998). Stability and control of a parametrically excited rotating system. part ii: Controls. Dynamics and control, 8(1), 19-35.

Boyd, S., El Ghaoui, L., Feron, E., and Balakrishnan, V. (1994). Linear matrix inequalities in system and control theory, volume 15. Siam.

Chen, C.T. (1998). Linear system theory and design. Oxford University Press, Inc.

Daqaq, M.F., Stabler, C., Qaroush, Y., and SeuaciucOsório, T. (2009). Investigation of power harvesting via parametric excitations. Journal of Intelligent Material Systems and Structures, 20(5), 545-557.

Kirkland, W.G. and Sinha, S. (2016). Symbolic computation of quantities associated with time-periodic dynamical systems. Journal of Computational and Nonlinear Dynamics, 11(4), 041022.

Löfberg, J. (2004). Yalmip : A toolbox for modeling and optimization in Matlab. In In Proceedings of the CACSD Conference. Taipei, Taiwan.

Mesquita, A.J.N. (2007). Análise da estabilidade de sistemas dinâmicos periódicos usando teoria de Sinha.

Peruzzi, N.J. (2005). Dinâmica não linear e controle de sistemas ideais e não-ideais periódicos.

Peruzzi, N., Chavarette, F.R., Balthazar, J.M., Tusset, A.M., Perticarrari, A.L.P.M., and Brasil, R. (2016). The dynamic behavior of a parametrically excited timeperiodic mems taking into account parametric errors. Journal of Vibration and Control, 22(20), 4101-4110.

Sinha, S. and Butcher, E.A. (1997). Symbolic computation of fundamental solution matrices for linear time-periodic dynamical systems. Journal of Sound and Vibration, 206(1), 61-85.

Sinha, S., Henrichs, J., and Ravindra, B. (2000). A general approach in the design of active controllers for nonlinear systems exhibiting chaos. International Journal of Bifurcation and Chaos, 10(01), 165-178.

Slotine, J.J.E., Li, W., et al. (1991). Applied nonlinear control, volume 199. Prentice hall Englewood Cliffs, NJ.

Sturm, J.F. (1999). Using sedumi 1.02, a matlab toolbox for optimization over symmetric cones. Optimization methods and software, 11(1-4), 625-653. 